\begin{abstract}
//Resumen:
Cambiar el concepto de dolor que se tiene del cementerio, de recuerdos de fecha o anécdotas oscuras que solo consiguen borrar la memoria, encontrando la mejor manera de olvidar la materia (edificación.) y los restos (ser), convirtiéndolos en centros oscuros de poca significación y mucho olvido; el mismo lugar encuentra la manera de recuperar la memoria Orientándola a lo físico, lo espiritual y Significativo dejándose utilizar como espacio pedagógico, como aula real para la recuperación tangible e intangible del patrimonio. Para encontrar la manera de revalorizar el sitio, se hace necesario el uso de herramientas pedagógicas efectivas que logren en el usuario crear una nueva visión, una catalogación e inventario, la difusión de los bienes patrimoniales y la re significación del cementerio. El Periplo Arquitectónico Funerario está conformado por estudiantes y docentes del programa de Arquitectura de la UAC, directivos del cementerio y propietarios de las tumbas; actividades como la visita guiada al lugar, la recolección de datos, la construcción del diagnostico socializado con los propietarios de las tumbas y la conceptualización a partir de un rompecabezas con la imagen de los elementos de patrimonio, dieron como resultado la re significación, re valorización del lugar por las personas participantes y la recuperación efectiva del patrimonio tangible e intangible.
\end{abstract}

\title{
//Abstract:
}

To approach a subject that it has had little study, in Cartagena, from the point of view of the architects, the study of the techniques, hardly he begins to blunt, with works that are base of the present I articulate, already have 20 years of use, unpublished, like global document, but with article publications of specific subjects, in which the work group has reunited experiences, from he practices it and the professional exercise of the restoration and conservation of the architectonic patrimony, from the study of other documentary sources, we must stake that this work had been impossible, without the direct perception of the buildings, that have allowed to identify with fullness when mudéjar in their essence. Thus the things, it undertook the investigation, on materials, techniques, that they determined the accomplishment of Cartagena of Indians, to write this I articulate, investigation project, use as it bases documentary the unpublished book "Techniques Old of Construction, the Permanence of the systems", by the authors of this communication, with the Architects Rosemary Martelo and Augusto Martinez of where they left most of the drawings, photography's.

\section{Resignificación de lugares funerarios. Resignificación de la visión del Cementerio Universal de Barranquilla [Salvador Coronado Cepeda]}

\author{
//Fecha de recepción [30 de mayo de 2011] \\ //Fecha de aceptación [1 septiembre de 2011]
}

\section{//Palabras clave:}

Revalorización, re conceptualización, Inventario, conservación, patrimonio.

\section{//Keywords:}


ARTE \& DISEÑO, ISSN: 1692-8555, Vol. 9 Núm. 2, Julio - Diciembre de 2011 Resignación de lugares funerarios. Resignación de la visión del Cementerio Universal de Barrranquilla, págs. 23-30

\section{EMOCIONES Y ARQUITECTURA A MANERA DE INTRO- DUCCIÓN}

Los espacios arquitectónicos son creados a partir de emociones en los procesos de articulación que se generan en el creador y desde la pericia del mismo; es así que la función en algunos casos, define al objeto, le da vida y significado en los usuarios, mas esta función utilitaria no garantiza su perdurabilidad.

Los objetos de arquitectura son creados con la finalidad de buscar en el usuario una aceptación emocional, comenzando con la percepción de la obra y terminando con la vinculación a ella desde lo sentimental, lo anterior es lo que garantizará en el tiempo la duración, perdurabilidad, mantenimiento y sostenibilidad del objeto arquitectónico, es así, como la apropiación del lugar se fortalece con la unión hombre-edificio, haciéndola parte del hombre para no dejarla morir y mantenerla en el tiempo. Se considera esta relación como el objetivo principal del arquitecto.

La intención entonces, de la arquitectura, es la comunicación de materia y sentimientos, articulados en las líneas, trazas horizontales y verticales que refuerzan los conceptos de la razón, aplicados a la intención de diseño, entonces, la razón y los sentimientos se mezclan en el objeto arquitectónico formando hitos de cultura y de significado en los usuarios.

Una vez encontrado el significado de la obra arquitectónica, el estudio de la función puede tener muchas acepciones como estéticas, formales, de uso, constructivas; al igual que los signos pueden tener muchos significados e interpretaciones. De la unión entre significado y función resulta un edificio que muestra la identidad de la comunidad de estudio.

Al hablar de las relaciones edificio-individuo u objeto-significado conseguidos en la arquitectura, creados conjugando perfectamente lo funcional, lo estético, lo racional; solucionando el problema de diseño, e interpretando la necesidad manifestada por los individuos, entendida por el arquitecto y suplida por el objeto de diseño; debemos referirnos a la significación e intención que el autor plasma en esta. Estos signos propios de la época en que se desarrolla el lugar, que con el uso de la edificación se le incorporan, este hecho da significado y carácter a la obra arquitectónica.

El significado en la arquitectura es entonces la interpretación real del problema de diseño con la manifestación y creación de un objeto arquitectónico intencional. Esta relación no se debe plantear aislada, hay que contextualizarla para darle efectividad al valor simbólico y el significado que se quiere conseguir al crear un objeto para ser disfrutado por el usuario y sus futuras generaciones.

\section{RAZONES PARA RE SIGNIFICAR SITIOS DE ARTE FU- NERARIO COMO ICONOS DE CULTURA}

Un icono funerario está dotado de historia, de edificios o bienes de interés patrimonial, elementos de arte, leyendas, costumbres; su desarrollo urbano muestra el desarrollo urbano de la ciudad, entendiendo la historia de la arquitectura de la ciudad a partir del sitio funerario.

Es la misma historia de la ciudad y de sus habitantes que crea estos espacios y los dota de obras de carácter singular haciéndolos únicos e irrepetibles, la ciudad de Barranquilla cuenta con un legado cultural al interior del Cementerio Universal, desconocido y desvalorado, no existe un significado con el cual remitirnos a este sitio, ni que el habitante pueda referenciar.

La problemática es de carácter nacional, y local, ya que los bienes de interés cultural que se encuentran en los cementerios están desfigurados e identificados sólo con sentimientos de temor, terror, pánico, escondidos en los cerramientos, deteriorados y subutilizados, con poco carácter simbólico y carentes de otros significados. En ellos se han olvidado los grandes próceres de la patria, escritores, literarios literatos, artistas y hasta los grandes urbanistas arquitectos que desarrollaron la ciudad, no existe señal del sitio fundacional del cementerio (la primera tumba, la tumba del fundador del cementerio).

La problemática se amplía cuando existen valores reconocibles y no se tiene protección legal sobre ellos.

Esta problemática esta también referida al contexto internacional, donde la pérdida de valores ha traído consigo el deterioro de los elementos que hacen parte de la antigüedad de los cementerios y la falta de mantenimiento, recrean en los usuarios lugares con todo tipo de problemas formales y edificatorios.

Las nuevas posiciones frente a la normatividad desfiguran las costumbres de los antepasados, para darle forma a la nueva legislación. La poca gestión con respecto a la protección, salvaguarda y tutela de los sitios funerarios los hace vulnerables, otro factor sumado a esta problemática, es el desconocimiento de las riquezas contenidas en el cementerio, la falta de personal preparado para abordar procesos de sensibi- 
lización, identificación, restauración, rehabilitación, revalorización histórica de los sitios funerarios, olvidando la dimensión humana y material, ocultando la identidad cultural.

Estas razones fundamentan una pregunta que trató de responder el taller de diseño VI orientado a la revalorización del Patrimonio Arquitectónico en los sitios funerarios.

¿Cómo encontrar en los sitios funerarios un significado que cambie la idea de sombra que tiene el individuo hacia los cementerios, logrando con ello la Resignificación de la visión del Cementerio Universal de Barranquilla?

\section{MEDIOS DE RESIGINIFICACIÓN-HERRAMIENTAS PARA RESIGNIFICAR}

Resignificar es encontrar en los hechos y acontecimientos nuevas posiciones o miradas objetivas de los mismos, es encontrar en la historia nuevas oportunidades para contarlas, no significa que se tenga que reescribir o escribir sobre ella, es mirarla desde otro punto de vista, más claro y objetivo. Es para nosotros encontrar un nuevo significado agregado al que se tenía, no se trata de cambiar de contexto las cosas, es contextualizarlas al momento actual, es encontrar el significado real de las cosas que han perdido su valor, su belleza, hasta reinterpretar, recorrer, revivir, revalorar, retomar los espacios y releer la historia con un nuevo punto de vista.

Entonces para la Resignificación de lugares se adoptan pautas desde la Programación Neurolingüística. Aquellas técnicas que tratan de cambiar la conducta en el ser humano con prácticas que le hacen comprender mejor las viejas situaciones convirtiéndolas en nuevas experiencias.

Una de estas técnicas es el rencuadre o resignificación, esta trata de cambiar marcos de referencia redefiniendo las situaciones experimentadas por el individuo, resultando un cambio de conducta y un cambio de posición. Esta herramienta de la Programación neurolingüística se toma y adapta a la experiencia que tienen los individuos con los sitios funerarios. Si el objetivo principal de la programación neurolingüística es la de modificar la conducta de los individuos mejorando los aspectos de situaciones vividas, para así, afrontar las nuevas, para la resignificación o rencuadre se necesita cambiar los mapas mentales invalidantes de la realidad por mapas mas potenciadores, es entonces que esta herramienta se válida para la resignificación de sitios funerarios, para el caso “Cementerio Universal de Barranquilla".
El uso de las técnicas de la programación neuro lingüística como la resignificación en la arquitectura, debe crear en los individuos una nueva visión o tratar de encontrar nuevos significados a la arquitectura que este usa. Esta herramienta, usada como generadora de conocimiento desde el currículo, acerca al estudiante a la realidad que están atravesando los sitios funerarios, una vez utilizada se valida con la apropiación de un nuevo significado, una nueva posición a lo que el estudiante llamaba cementerio "los cementerios son la puerta para analizar la arquitectura a través de la historia, marcan un periodo y situaciones especificas de una población. Es un sitio de valor incalculable de arquitectura, es una joya arquitectónica. Son lugares tranquilos. Ambientes agradables que contribuyeron al desarrollo de la ciudad. Se cambia totalmente la percepción con una idea de calma y fuera el caos de la ciudad", algunas de las percepciones del taller de Diseño VI, después del periplo arquitectónico al Cementerio Universal de Barranquilla.

Cobra entonces la importancia de que el currículo articule las necesidades de la ciudad y sus habitantes, orientado a sustentarse en los pilares de la educación, aprender a conocer las diversas teorías y maneras de adentrarse en procesos de restauración, vigilando la aplicación de las normas para su conservación, salvaguarda, tutela y uso. Aprender a ser, o la manera como el estudiante demuestra las habilidades y destrezas adquiridas en el aula, trasladándolas al estado actual que presenta el patrimonio arquitectónico (competencia específica). Aprender a ser un individuo que detecta la situación y se compromete con la comunidad y trabaja con ella y para ella (responsabilidad individual).Aprender a convivir desde la responsabilidad social, generando valores y el respeto a las diferencias. El currículo flexible que se maneja al interior del Taller de Diseño VI, permite la interacción permanente estudiante-docente, este currículo centra su función desde lo dialógico, orientado a la solución de la problemática de la comunidad detectada por la institución y manifestada por ella.

Otra herramienta de apropiación por parte del taller al estudiante es el manejo de la normativa internacional, como lo son las cartas de restauración monumental, incluyendo en este ejercicio la carta de Morelia referida a los cementerios patrimoniales y el arte funerario.

Estos documentos son manejados por los estudiantes como cartas de navegación para el manejo, difusión, conservación, restauración, mantenimiento y reparación, tendientes a asegurar la salvaguardia tutela y uso de los bienes de interés cultural en sus ámbitos. 
Estas cartas facilitan la vinculación de los demás cursos, como lo son el curso de investigación y el de urbanismo trabajando conjuntamente en lo pluridisciplinar.

La educación en Colombia facilita estas actividades pedagógicas tendientes a la preservación, intervención del patrimonio, desde la normativa y específicamente la resolución emanada en el Ministerio de Educación Nacional, que reglamenta las condiciones mínimas de calidad y los requisitos para el ofrecimiento y desarrollo de programas académicos de educación superior, asegurando las competencias cognitivas que permitan al estudiante desempeñarse en los campos de la intervención patrimonial, la valoración, conservación, el manejo del patrimonio local, regional y nacional.

El sustento de la estrategia pedagógica como herramienta para la resignificación de lugares, se consigue con la operacionalización de un currículo flexible, orientado a la participación activa de todos sus miembros, creando formas de participación y difusión de conocimientos, encontrando vínculos rotos, originando formas de construcción y utilizando técnicas para reforzar y construir identidad, encontrando, significando y dando afecto a los lugares. El dicente encuentra entonces símbolos que permiten identificarse y resignificarse dándole sentido al uso actual que tiene el cementerio, encontrando en la belleza, lo arquitectónico, lo urbano nuevos significados, recreando nuevos símbolos que permitan construir y refuercen nuestra identidad individual y colectiva resultando la resignificación de los lugares.

Por último, la construcción de significados en la pedagogía acerca al educando a la cultura y poco apoco a conseguir la construcción de significados a partir de los conocimientos y experiencias que se tengan con relación al tema, es la educación la que se encarga de este acercamiento, es la construcción de significados expuesta por J. Bruner lo que facilita la creación de nuevos significados en el concepto previo del cementerio, es revalidar el concepto anterior, explorando nuevas formas de adquirir el conocimiento, construyendo significados e ir más allá del término. El acercamiento a la cultura funeraria, la interpretación, el reconocimiento y la difusión está ejerciendo un proceso de creación de nuevos significados, con el constante cambio del significado y la posibilidad de recrearlo o resignificarlo nuevamente.

\section{RESIGNIFICACIÓN DE LUGARES}

Para arremeter un ejercicio de resignificación de lugares, debe confrontarse al individuo con un pasado o hecho histórico, para así, recomenzar su nueva posición frente al hecho actual o el renacimiento del hecho histórico con otra visión. Se trata de remirar objetivamente al lugar desde otros puntos de vista, legales, estéticos, sociales, culturales, arquitectónicos, etc.

- Etapas de confrontamiento.

Para responder ante el hecho de la resignificación de lugares se deben suplir una serie de etapas, que consigue enfrentar la posición actual del individuo con la construcción de nuevos significados

- Objetividad Cultural.

La estrategia pedagógica acerca al estudiante a la cultura del lugar y la construcción de nuevos Símbolos, para reforzar la identidad, una vez se conoce el sitio desde otra visión se identifica al Cementerio como un ente culturizador o lleno de cultura donde el individuo recrea, recorre, usa la nueva visión y la hace suya.

- Nueva Obra / Nueva Posición.

Reconceptualización de la visión del lugar a partir de nuevos esquemas mentales construidos por ellos con la ayuda de la fotografía, la lúdica y los nuevos símbolos que se adhieren al concepto anterior, el lugar ya no es solitario y triste, es un lugar que se recorre y se vive, con grandes valores arquitectónicos y culturales.

- Resurgir de los valores.

Con el acercamiento cultural al lugar el individuo crea nuevos mapas mentales o esquemas de pensamiento con relación al lugar funerario, estos esquemas son resignificados con la belleza, lo arquitectónico, urbano, histórico, social, legal que se le suman al concepto inicial enriqueciéndolo y resinificándolo. Esta resignificación está centrada en la resignificación social, económica y cultural.

- Tasación de valores.

La valoración se consigue con la objetividad de la relación que existe entre el sujeto y el objeto, tasando los valores materiales e inmateriales de igual forma. El ejercicio de la valoración orienta los procesos de catalogación y repercuten en la recuperación y formación de identidad creando una estrecha relación objeto-sociedad, el objeto se convierte en un signo de valor con el solo hecho de ser estudiado. La escala de valores plantea una relación objeto-sujeto y contexto, los criterios de valoración objetivos 
propenden por un inventario que valora, protege los atributos contenidos en los bienes de interés cultural, tendrán en cuenta los valores culturales, estéticos, simbólico e histórico, resaltando el hecho de que estos son contenedores de otros. La conjugación de los valores contenidos en el bien los califica como bien de interés cultural, permitiendo a la sociedad que los identifique y los signifique culturalmente.

- Resignificar la historia y la memoria del lugar. Encontrar los vínculos perdidos por el paso del tiempo ayuda en la historia a resignificar los lugares, ellos están ligados a los acontecimientos y son testigos de la historia la escriben y también la ocultan, los vínculos entre la comunidad y el objeto funerario se fortalecen cuando la historia actual lo permite, la significación que le da la sociedad a sus símbolos los hace perecederos permite su apropiación y adopción, para ser salvaguardado, tutelado y usado. Entonces la historia no se debe borrar, se debe encontrar en ella una objetividad para ser nuevamente vista con una visión actual.

\section{ESTRUCTURA DE LA ESTRATEGIA PEDAGÓGICA}

El propósito de la estrategia pedagógica es acercar al estudiante a la realidad de los cementerios, aprovechando los recursos para la catalogación, inventario, difusión, tutela y uso del bien de interés cultural presentes en el cementerio universal de Barranquilla. Este espacio pedagógico se convierte en fuente de conocimiento y ejercicio para el desarrollo de habilidades y destrezas adquiridas en el aula y en las diferentes etapas de formación profesional. Conjuga los saberes de la educación Ser-Hacer-Conocer- Convivir, a través del análisis crítico de los elementos patrimoniales estudiados catalogándolos y valorizándolos de acuerdo a sus características individuales y colectivas que favorezcan su protección y resguardo.

Apoya el proceso de apropiación y revalorización del espacio funerario desde la memoria y lo simbólico, termina en la difusión, tutela y uso de los bienes de interés cultural por parte de las personas que están en contacto permanente con el lugar, además, de contribuir a resaltar su riqueza estética, arquitectónica, urbanística, histórica y su inmenso valor como elemento generador de memoria, identidad y símbolos, creando en la ciudad un nodo cultural, memorial y de actividades urbanas de carácter público.

\section{PERIPLO ARQUITECTÓNICO}

Resignificación de la visión del cementerio universal de barranquilla Entrega de encuesta. Debe ser diligenciada antes de entrar al lugar.

- Sesión 1

Visita Guiada Encuentro con la historia: se realiza por un miembro del cementerio, para familiarizar al estudiante con el lugar.

- Sesión 2

Reparando la historia: Conceptualización Es la etapa más significativa e importante, se procede a la entrega de un rompecabezas de uno de los elementos arquitectónicos más importante y con los que el participante pueda rememorizar, recordar y revivir una nueva experiencia agradable dentro del lugar. Este rompecabezas es la imagen de uno de los elementos más significativos del lugar que en su parte posterior tiene palabras claves, como, vida, fabulas, historias, leyendas, colores, alegría, luz, memoria, ilusión, etc.

- Sesión 3

Huellas del lugar: Toma de datos. Observación directa. Iniciar la toma de datos directa sobre el elemento patrimonial, para proceder a su catalogación Toma de datos se procede a:

Exploración de datos físicos. Recolección de datos constructivos. Incidencia de procesos ambientales. Diligenciamiento de la ficha de inventario de bienes culturales inmuebles. Formato Ministerio de Cultura

\section{- Sesión 4}

Propuesta de recorrido para el lugar. Resignificación de la historia: Recorrido indicando en la planimetría el valor histórico de los elementos patrimoniales, el proceso de evolución urbana y artístico arquitectónico del lugar, señalando los periodos de crecimiento.

- Sesión 5.

Propuesta de intervención. Revalorización del bien Propuesta de intervención sobre el bien de interés cultural asignado al estudiante para su estudio mausoleo, tumba, monumento, vía, etc.

- Sesión 6.

Documento diagnostico. Es la recolección de evidencias de la estrategia pedagógica que puede ser utilizada para la catalogación e inventario del sitio funerario para su inclusión en la lista de los bienes de interés cultural en cualquiera de sus ámbitos, además, también puede ser utilizado por los propietarios de los bienes para arremeter procesos de restauración en sus bienes 


\section{- Sesión 7}

Taller de revalorización con familiares. Trata de resaltar la importancia que tiene su bien en el contexto del sitio cultural, el cual debe ser conservado para el acervo cultural de la ciudad.

\section{ANOTACIONES DE LOS ESTUDIANTES}

La intencionalidad de la presente investigación formativa, es la integración de pautas de la sicología social, la pedagogía, la programación neurolingüística y la arquitectura, consiguiendo a través de la estructuración de una estrategia pedagógica tendiente a la resignificación de lugares. Para este fin se utilizaron métodos de recolección de datos como la encuesta, construida con criterios de exploración a partir de criterios de significación planteados por Jerome Bruner.

Con relación a los planteamientos expuestos por este como:

- La exploración de los sentidos: se pregunta sobre la importancia de los cementerios.

- Los estados intencionales: para encontrarlos sentimientos que le puede generar el sitio.

- Los símbolos: para indagar si el encuestado identifica al lugar.

- Los referentes culturales: para saber que conoce de la cultura del lugar.

Entonces, las preguntas se organizan de la siguiente manera:

1. En una escala de valores de uno a cinco, siendo uno el valor más alto, cite el lugar que ocuparía el cementerio en la ciudad. Con esta pregunta se indaga sobre el conocimiento de uso que se tiene de la edificación su importancia y relevancia frente a otras edificaciones de la ciudad que forman parte del equipamiento urbano.

A este cuestionamiento los encuestados el 75\% manifiesta que el grado de importancia es bajo dándole una calificación de uno, el 25\% restante le da una importancia de tres.

2. Cuál es la percepción que tiene al interior de un cementerio o cerca de este. Al indagar sobre los sentimientos de los estudiantes muchos coincidieron en que son lugares oscuros y con significados como soledad, miedo, tristeza, dolor, peligro, respeto, tranquilidad, abandono, frio. Esta pregunta sirve para la verificación de los esquemas mentales o estados emocionales de los encuestados frente al lugar.
El sentimiento que registró el $58 \%$ fue la soledad, mientras que para sorpresa, el miedo o temor solo registro el $16 \%$ al igual que el frio, la tristeza y el dolor, mientras que el abandono registró el $41 \%$.

3. Existe una relación entre el proceso evolutivo de la ciudad y el desarrollo del cementerio. Este cuestionamiento pretende indagar sobre si el cementerio es un símbolo o forma parte de los símbolos de la ciudad y que si formo parte del desarrollo de la ciudad en este aspecto los encuestados le asignaron un porcentaje del $83 \%$ al concepto de que si forman parte importante en el desarrollo de la ciudad.

4. Considera usted que existe un valor artístico al interior de los cementerios. La pregunta cuestiona sobre la cultura o el conocimiento de la cultura funeraria del encuestado, donde el total de los encuestados considera que al interior de los $(\dot{i})$ existe grandes valores arquitectónicos, esculturas en sus tumbas.

5. Cuál cree usted es el origen de los cementerios.

Al igual que la pregunta anterior está dirigida a saber el grado de cultura tienen los encuestados de los cementerios Los encuestados en general dicen que el origen de los cementerios se da por una necesidad de la ciudad de enterrar sus muertos.

\section{CONCLUSIÓN GENERAL}

Esta herramienta pedagógica facilita la apropiación de los bienes de interés cultural creando nuevos significados en los sitios funerarios, adoptando nuevas posiciones en los esquemas mentales, resignificando los conceptos, creando significados destinados ser transmitidos y resignificados, apropiándose de leyendas, historias, nuevos conceptos de arte y arquitectura. Terminando con la construcción y resignificación de los nuevos sitios funerarios, conservados, tutelados y usados por una comunidad que se identifica individual y colectivamente con estos signos.

El presente trabajo creó, adaptó y utilizó una herramienta pedagógica para la resignificación de lugares funerarios apoyados por el acercamiento del currículo a la realidad de la comunidad, como base para la introducción a la vida laboral de los estudiantes del taller de Diseño VI, además, se convirtió en una manera de lograr la difusión del patrimonio arquitectónico en los lugares funerarios.

Una vez terminada las sesiones de la estrategia pedagógica, se registró por escrito la nueva visión que tienen los participantes del periplo arquitectónico en el cementerio universal de Barranquilla. 
Los comentarios se centraron en la nueva visión después del acercamiento al acervo cultural del lugar, concluyen lo siguiente:

- Antes consideraba al cementerio un lugar solitario y terrorífico, una vez entre se cambia totalmente la idea se siente una calma fuera del caos de la ciudad.

- Al ingresar al cementerio la percepciones es diferente, agradable, en el recorrido guiado por el funcionario se identificaron mausoleos importantes de personas que contribuyeron al desarrollo de la ciudad, sucesos históricos, además de ser un lugar tranquilo y fresco.

- Hay mausoleos importantes con un diseño arquitectónico que los hace únicos y predominan, a su vez le dan una importancia y representación al cementerio.

- La percepción que ahora tengo del cementerio es que es un lugar donde hay mucha historia y sus tumbas son una joya arquitectónica valiosa.

- Pienso que los cementerios más que un lugar donde se dejan los seres queridos una vez mueren, encontramos la historia de la ciudad, diseños exclusivos para cada bóveda, historia de la arquitectura, estilos y vegetación.

- Los cementerios funcionan como una puerta para analizar la arquitectura a través de la historia, marcan un periodo, unas técnicas y situaciones específicas de una población.

\section{RECOMENDACIONES}

- La educación ofrece herramientas para la salvaguardia, conservación, tutela y uso de los bienes de interés cultural, para vincular a la comunidad y trabajara en la difusión del patrimonio y su catalogación, dando como resultado el fortalecimiento de la identidad individual y colectiva.

- Los procesos de catalogación se deben iniciar en la educación media, para al llegar a la formación profesional la catalogación se convierta en un proceso efectivo de conservación de los bienes de interés cultural.

La intervención en los bienes de interés cultural se deben realizar por profesionales expertos con la vigilancia de las instituciones estatales.

- Los procesos de catalogación al interior de los lugares funerarios tienen que tener la participación de todos los actores del proceso, propietarios, funcionarios, expertos y la comunidad.
- La vigilancia de la aplicación de las medidas legales de protección de los bienes de interés cultural se debe articular con los reglamentos y normas de los lugares funerarios.

- La educación debe centrar su objetivo en construir identidad, además, de brindar las herramientas necesarias para las competencias del hombre para su uso en la sociedad; esta construcción se debe iniciar con la difusión del patrimonio material e inmaterial de las comunidades, teniendo en cuenta la memoria y la historia.

- El patrimonio es individual y de diversos valores es deber de todo ciudadano conservarlo, tutelarlo y usarlo, por esta razón se hace necesario la salvaguardia de estos a partir de su catalogación e inventario.

- La difusión efectiva de las manifestaciones culturales de los lugares funerarios se debe hacer de la mano de procesos de resignificación con la colaboración de la comunidad, las entidades estatales, escuelas.

- La inclusión de la cátedra de patrimonio en los currículos escolares de la educación media, para el acercamiento de los dicentes a las manifestaciones culturales de su comunidad.

- Debe hacerse por parte de los sitios funerarios un trabajo de acercamiento de sus manifestaciones culturales a la comunidad para su apropiación y significación.

- Crear en los sitios funerarios una subvención para aquellos monumentos que presentan procesos de deterioro.

- Valerse de las distintas entidades que pueden arremeter un proceso de catalogación e inventario en los bienes de interés cultural de los sitios funerarios para su salvaguarda y recuperación.

- Trabajar de la mano con la comunidad creando programas que faciliten el acercamiento de los individuos al cementerio, facilitando la apropiación y el fortalecimiento de la identidad, resultando la construcción de nuevos significados o la resignificación de los cementerios.

\section{BIBLIOGRAFÍA}

Renato de Fusco, La Idea de Arquitectura, editorial Gustavo Gili S.A. Barcelona 1976

D. Medina Lasansky. Brian Mc Laren. Arquitectura y Turismo. Editorial Gustavo Gili. S.A. Barcelona, 2004. 
Francisco de Gracia, Construir en lo Construido, La arquitectura como modificación, Editorial Nerea, Guipúzcoa, España, 2001.

Aldo Rossi, Para una arquitectura de tendencia, Gustavo Gili S.A. Barcelona, 1975

Rudolf Arnheein, La forma visual de la Arquitectura, Gustavo Gili S.A. Barcelona, 2001.

Josep Montaño la Thornberg, La arquitectura como lugar, colección arquitectura y critica. Gustavo Gili, Barcelona, 1974.

Salvador Carrión, Programación Neurolingüística, océano, Barcelona, 2006.
CARTA DE ATENAS, Carta internacional sobre la conservación de los monumentos de arte e historia. 1931.

CARTA DE VENECIA, Carta internacional sobre la conservación y la restauración de los monumentos y los sitios. 1964

CARTA DE BURRA, Carta internacional de ICOMOS para Sitios de Significación Cultural. 1999.

CARTA INTERNACIONAL DE MORELIA, Carta internacional relativa a cementerios patrimoniales y arte funerario. 2005

Dirección de Patrimonio, Manual para el inventario de bienes culturales inmuebles, Ministerio de Cultura, Bogotá D.C. 2005 